\title{
Interplay of Polarity Proteins and GTPases in T-Lymphocyte Function
}

\author{
Ivan Fung, ${ }^{1,2}$ Sarah M. Russell, 1,2,3 and Jane Oliaro ${ }^{1,2}$ \\ ${ }^{1}$ Cancer Immunology Program, Peter MacCallum Cancer Centre, East Melbourne, VIC 3002, Australia \\ ${ }^{2}$ Department of Pathology, The University of Melbourne, Parkville, VIC 3010, Australia \\ ${ }^{3}$ Centre for Micro-Photonics, Swinburne University, Hawthorn, VIC 3122, Australia
}

Correspondence should be addressed to Jane Oliaro, jane.oliaro@petermac.org

Received 15 November 2011; Accepted 13 December 2011

Academic Editor: Alexandre S. Basso

Copyright ( $) 2012$ Ivan Fung et al. This is an open access article distributed under the Creative Commons Attribution License, which permits unrestricted use, distribution, and reproduction in any medium, provided the original work is properly cited.

\begin{abstract}
Polarity refers to the asymmetric distribution of different cellular components within a cell and is central to many cell functions. In T-cells, polarity regulates the activation, migration, and effector function of cytotoxic T-cells (CTLs) during an immune response. The regulation of asymmetric cell division by polarity proteins may also dictate CTL effector and memory differentiation following antigen presentation. Small GTPases, along with their associated polarity and adaptor proteins, are critical for mediating the polarity changes necessary for T-cell activation and function, and in turn, are regulated by guanine exchange factors (GEFS) and GTPase activating proteins (GAPS). For example, a novel GEF, dedicator of cytokinesis 8 (DOCK8) was recently identified as a regulator of immune cell function and mutations in DOCK8 have been detected in patients with severe combined immunodeficiency. Both B and T-cells from DOCK8 mutant mice form defective immunological synapses and have abnormal functions, in addition to impaired immune memory development. This paper will discuss the interplay between polarity proteins and GTPases, and their role in T-cell function.
\end{abstract}

\section{Overview of Polarity}

Polarity refers to the asymmetric distribution of surface receptors, cytoskeletal components, vesicle trafficking, and signaling proteins within a cell [1]. Many polarity components are conserved between different cell types and organisms (reviewed in [2]). Polarity is an important factor in T-cell functions, such as immunological synapse (IS) formation, migration, target cell killing, asymmetric cell division (ACD), and differentiation [3-8]. In order to establish and maintain polarity in response to dynamic cellcell interactions and extracellular cues, a T-cell must be able to orchestrate different signals to regulate the different recruitment of many cellular components. This process is highly regulated and involves both GTPases (reviewed in [9]) and a network of polarity proteins [1].

GTPases act as molecular switches to control cellular processes. The family of Rho GTPases includes Cdc42, RhoA, and Rac1 (reviewed in [10]). GTPases have two conformational states, which are dependent on the type of guanine nucleotide bound. The active state is induced by the binding of Guanosine- $5^{\prime}$-triphosphate (GTP), and the inactive state is induced when Guanosine diphosphate (GDP) is bound. The loading of GTP and dissociation of GDP are regulated by different proteins: guanine exchange factors (GEFs) promote the exchange of GDP for GTP, GTPase activating proteins (GAPs) catalyze the activity of GTPase activity to their downstream effectors, and the guanine nucleotide dissociation inhibitors (GDIs) block regulation (reviewed in [11]). Activated Rho GTPases regulate cytoskeleton remodeling, which in turn influences morphology, migration, and protein trafficking (reviewed in [12]). Like other members of the Rho GTPase family, Cdc42 influences a large array of cellular activities. Its downstream effectors include a large number of kinases which activate many signaling pathways $[13,14]$ as well as nonkinase proteins, such as neuronal Wiskott-Aldrich Syndrome protein (N-WASP) [15] which promotes actin nucleation.

The evolutionarily conserved polarity proteins are localized into different regions of a cell to act as scaffolds for the 
recruitment of other protein complexes (reviewed in [16]). The Scribble, Par and Crumbs polarity protein complexes are the most extensively studied. The Scribble complex consists of Scribble (Scrib), Discs large (Dlg), and Lethal giant larve ( $\mathrm{Lgl}$ ) proteins (reviewed in [17]). The Scribble and Par complexes regulate asymmetric cell division (ACD) of neuroblasts in Drosophila (reviewed in [18]). The Par complex, which consists of Par3, Par6, and atypical protein kinase C (aPKC), was first discovered in C. elegans embryos that have defective anterior-posterior partitioning [19]. The Crumbs complex consists of Crumbs, PatJ, and Pals1 (reviewed in [2]) and is important in mammalian epithelial cell polarity [20]. All of these proteins, with the exception of aPKC, consist of a variable number of binding motifs termed PSD-95/Dlg/ZO-1 (PDZ) domains [2]. The PDZ domain can interact with a number of signaling proteins; for example, Dlg1 can interact with protein tyrosine phosphatase and tensin homologue (PTEN) [21] as well as with other PDZ-containing proteins and the Par6-aPKC complex can interact with Lgl, Par3, and Pals1 (reviewed in $([1,2])$. Polarity proteins establish a network to orchestrate signals throughout the cell in response to extracellular cues. The polarity proteins can work cooperatively or antagonistically [17] to regulate cell polarity. Polarity proteins also work in conjunction with GTPases to establish and maintain cell polarity (reviewed in [22]).

\section{Polarity in T-Cells: The Immunological Synapse}

Two main classes of T-cells are produced after maturation and selection in the thymus: $\mathrm{CD} 8^{+} \mathrm{T}^{-}$-cells and $\mathrm{CD} 4^{+} \mathrm{T}$ cells, distinguished by their expression of either the cell surface marker, cluster of differentiation 8 (CD8) or 4 (CD4). $\mathrm{CD}^{+} \mathrm{T}$-cells function as cytotoxic T-lymphocytes (CTL) and have the ability to kill target cells, such as virus-infected cells, by releasing pore-forming perforin and serine protease granzymes via exocytosis [23]. To carry out their immune functions, CTLs must first be activated. CTL activation involves the interaction of the T-cell receptor (TCR) with pathogen-derived peptide antigen presented by antigen presenting cells (APCs) via their major histocompatibility complex (MHC) class I molecule. An immunological synapse (IS) is formed when a TCR interacts with peptide MHC (as reviewed in $[24,25]$ ). T-cell activation also involves an important second signal, which is provided by the interaction between the costimulatory molecules on the T-cell and APC. The importance of the co-stimulatory signal in naive T-cell activation has been demonstrated in many in vitro studies (reviewed in [26]).

During IS formation, many molecules and complexes are recruited towards, or away from, the cell-cell interface. Molecules such as the TCR and microtubule organizing centre (MTOC) are recruited to the interface, while CD43, a member of sialoglycoproteins, is polarized to the distal pole, away from the interface [27]. At the interface, compartmentalization of proteins was first described by Kupfer's group, where surface molecules are clustered to regions termed supramolecular activation clusters (SMACs) [28]. In a mature IS, the central region, or CSMAC, contains the TCR, CD28, and their associate signaling molecules. The cSMAC is surrounded by an outer ring of adhesion molecules including lymphocyte function-associated antigen 1 (LFA1 ), and talin, a cytoskeleton protein that links integrins to the actin cytoskeleton [29,30]. The formation of the IS is a dynamic process. Initial antigen-independent contacts between the T-cell and the target cell involve the interactions of adhesion molecules such as CD2 with LFA-3 [31] and LFA-1 with ICAM-1 [28]. LFA-1 and ICAM-1 localize to the CSMAC and TCR-MHC complexes to the pSMAC at the initial phase of IS formation. However, in a mature IS the situation inverts and the TCR-MHC complex resides in the cSMACs, while the antigen independent interactions are at the periphery [24]. An important implication of a polarized and compartmentalized IS is the regulation of T-cell activation, by controlling TCR signaling and TCR degradation [32]. Studies have shown that the CSMAC plays a role in TCR degradation in the event of strong agonist interactions [33], and it has been proposed that signals from weaker interactions are enhanced [34]. The exact role of the synapse is still controversial $([35,36])$, however, these studies highlight the importance of the polarized and compartmentalized nature of the immunological synapse.

While the nature and function of the TCR-MHC complex has been intensively studied, the role of LFA-1 and its interaction with its ligand, ICAM-1, in the pSMAC has only recently been elucidated. LFA-1 is part of the large family of leukocyte integrins and is expressed on $\mathrm{T}$ and $\mathrm{B}$ lymphocytes. It is involved in a wide range of $\mathrm{T}$-cell functions including activation upon antigen presentation, CTL-mediated killing, cell adhesion, and migration. The importance of LFA-1 in the immune system is highlighted by patients with leukocyte adhesion deficiency (LAD) who have impaired pathogen clearance and suffer repeated infections [37]. Integrin $\beta 2$ chain (CD18)-deficient mice displayed defects in leukocyte adhesion and proliferation [38]. LFA1 is critically involved in the initial contact of a T-cell with the APC [39]. This contact is essential for T-cell activation as it provides the stop signal for a migrating T-cell to scan the surface of the APC for peptide-MHC. The TCR-peptideMHC interaction activates LFA- 1 and increases its affinity and avidity, resulting in a stringent interaction with its ligands, such as ICAM-1 (reviewed in [40]). This stronger interaction is believed to be a stabilizer in T-cell dendritic cell (DC) interactions [41] therefore allowing sustained TCR signaling. LFA-1 is also needed for Erk1/2 signaling during antigen presentation [42]. The Erk1/2 signaling pathway promotes T-cell activation and proliferation. LFA-1 is one of the many proteins that regulate IS formation and, as discussed above, is critical for normal T-cell activation and proliferation.

To carry out its highly specialized functions, the IS and its associated signaling and adhesion proteins are tightly regulated. The change in morphology that occurs when a Tcell contacts a target cell is mediated by actin cytoskeleton rearrangement. TCR signaling induces phosphorylation of myosin II [43], which causes loss of myosin filaments [44]. This allows for the depolymerization of the actin 
cytoskeleton in the midbody, and in the uropod, facilitating change in morphology. The Scribble complex is also believed to be involved in myosin II regulation [45]. Scribble and Dlg are transiently recruited to the cell-cell interface upon IS formation $[6,46]$. TCR signaling induces dephosphorylation of pERM, which leads to relaxation of the cytoskeleton, allowing Scribble and Dlg to be recruited to the synapse. This process is mediated by cytoskeleton rearrangements that are regulated by Rho and Rac GTPases [47]. TCR signaling also leads to Vav-(a GEF for Cdc42) mediated cytoskeleton remodeling. After activation by TCR signaling, Vav activates Cdc42 and Rac1 [48, 49], which in turn activates WASP and PAK. WASP promotes actin nucleation, which generates a contracted actin network that serves as a scaffold for signaling molecule recruitment. Scribble may recruit Rac1 and Cdc42 to the IS through the p21-activated kinase [PAK]-interacting exchange factor ( $\beta$-PIX) [50] and may bring the GTPases into close proximity to their downstream effectors and many signaling molecules (Figure 1(a)). $\beta$ PIX and Scribble have been shown to interact in other cell types, so this interaction may also provide a mechanism for recruitment of $\beta$-PIX to the IS following TCR stimulation. TCR signaling also leads to the activation of downstream transcription factors, which play a major role in regulating asymmetric cell division and differentiation, and polarity proteins may serve as an integrating platform for various signals.

\section{Polarity in T-Cells: Asymmetric Cell Division}

As discussed above, a naïve T-cell is activated after interacting with the peptide-MHC molecule on APCs, during which an IS is formed. Differentiated cells are well-characterized by the expression levels of specific cell surface markers and immune functions, as well as transcriptional events in the developmental pathway. However, there are competing hypotheses on the mechanism that gives rise to the large variety of functionally diverse subsets of T-cells [51-56]. In the "one cell, one fate" model, naïve cells are activated after receiving unique signals and give rise to a homogeneous populations of progeny cells. The generation of different subsets of cells is therefore determined by factors such as antigen availability over time and degree of maturation of the DCs [57]. The "one cell, multiple fates" model proposes that naïve cells undergo asymmetric cell division after activation and give rise to two daughter cells that are committed to different cell fates, thus generating a heterogeneous population of progenies [58]. Asymmetric cell division involves the establishment of an axis of polarity, which may be influenced by different external cues such as the microenvironment and the orientation of the mitotic spindle to the axis. Fate determinants are recruited into the two daughter cells and after division, each daughter cell inherits a different set of determinants, which set them on different paths of cell fate (reviewed in [59]). Asymmetric cell division has been observed in different cell types in mammalian cells [60] and is evolutionarily conserved across many organisms. One example is when a Drosophila sensory organ precursor (SOP) undergoes asymmetric cell division to produce a pIIa cell and pIIb cell. Following another round of asymmetric cell division, the pIIa daughter cell gives rise to a socket and a shaft granddaughter cell. One of the daughter cell of pIIb is programmed to die, while the other gives rise to a neuron and a sheath cell [61].

The first evidence to show that asymmetric cell division occurs in T-lymphocytes was reported by Chang et al. [5]. This study, and others since, has shown that polarity proteins, cell fate determinants such as Numb, and transcription factors, are asymmetrically distributed in T-cells during cell division $[4,5,8]$. Most interestingly, the putative "proximal" daughter cells (isolated by high expression of CD8) provided acute, but poor long-term, protection against Listeria infection after adoptive transfer of the daughter cells into recipient mice. In contrast, the low CD8 expressing daughter cells (putative "distal" daughters) gave long-term protection [5]. Scribble, aPKC and Par3 are all asymmetrically distributed during cell division in T-lymphocytes. However, the mechanisms of ACD, as well as how extracellular cues, such as the degree of DC maturation and the cytokine environment, can influence asymmetric cell division and ultimately cell fate, are poorly understood.

\section{Polarity in T-Cells: Migration}

Migration is particularly important in the context of Tcell activation and effector functions, as T-cells undergo a number of scanning steps before antigen recognition. When a T-cell migrates, it establishes a front-rear polarity with a leading edge and a trailing end (reviewed in [3, 62]). The leading edge of the cell, or lamella, has a high concentration of free actin filaments to generate contractile force [3], and chemokine receptors such as CCR2 and CCR5 [63] to facilitate effective homing of the lymphocyte. The posterior of the cell contains a protrusion, termed the uropod, which adheres to the substratum, allowing the lymphocyte to move forward (reviewed in $[27,45]$ ). The MTOC, the TCR, ezrin, and adhesion molecules, such as CD43, and intercellular adhesion molecule-1 (ICAM) [64] are polarized to the uropod. GTPases and polarity proteins regulate the spatial organization of these cellular components.

The shape of a migrating $\mathrm{T}$-cell is dynamic and requires continual rearrangement of the actin cytoskeleton in the lamellipodia [65]. Therefore, a T-cell must be able to remodel its cytoskeleton efficiently. GTPases are central to this process and the spatial regulation of their activity enables cell movement and controls directionality (reviewed in [66]). Rac1 and Cdc42 promote actin nucleation at the leading edge of a T-cell via WASP and Scar proteins, which induce Arp 2 and Arp3 proteins to bind to actin monomers and promote nucleation. Nucleation of actin monomers catalyzes actin polymerization $[1,67]$. Racl promotes protrusion and Cdc42 induces filopodia. Cdc42 is also essential for directing a migrating cell to extracellular cues [68]. Another important GTPase is RhoA. Activation of RhoA is required for uropod formation. ROCK protein kinase is one of the downstream effectors of RhoA [69]. ROCK signaling results in cell body contraction and rear end retraction (reviewed [1]). 


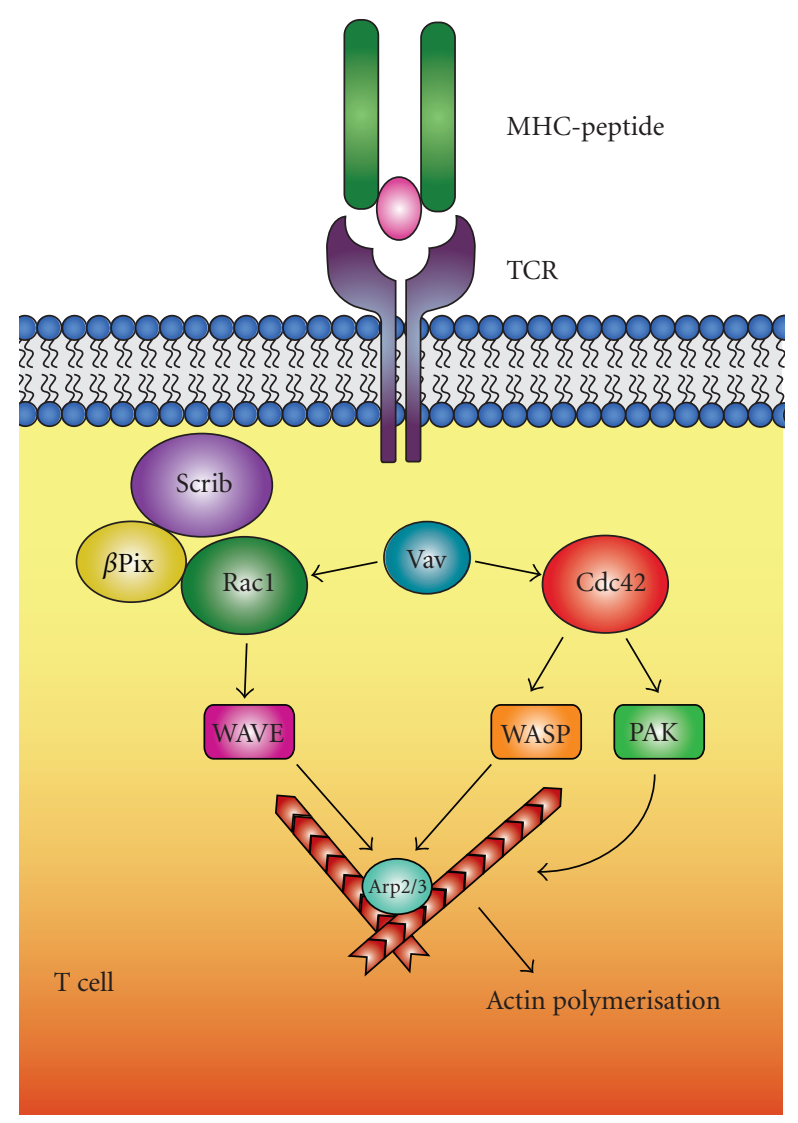

(a)

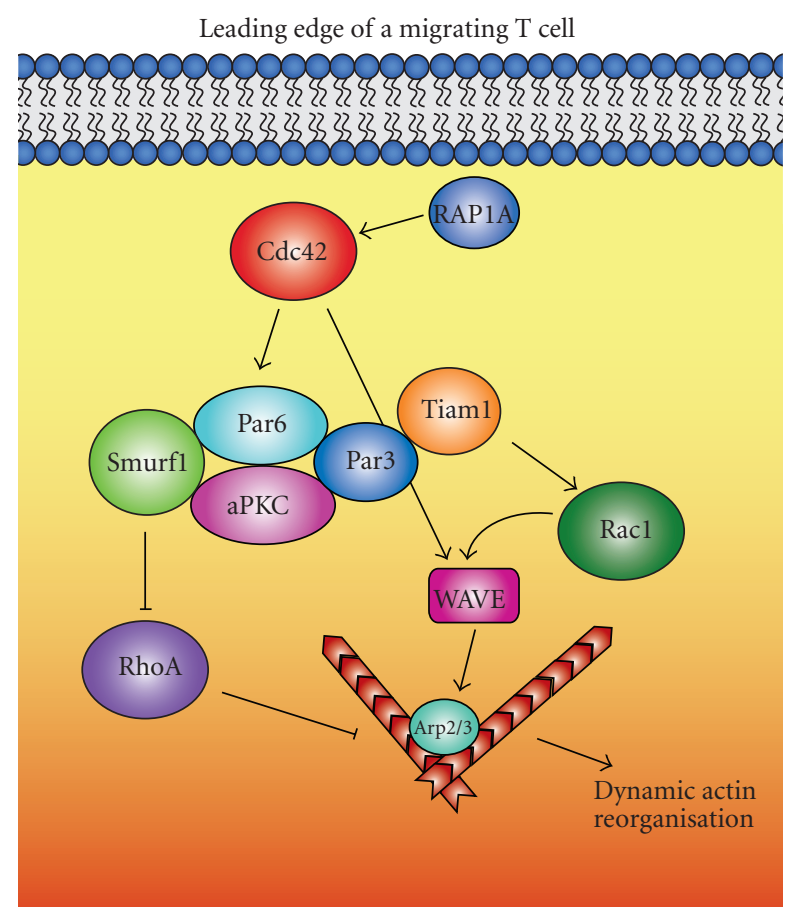

(b)

Figure 1: GTPases are important mechanical switches in T-lymphocyte function. (a) During antigen presentation, a T-cell undergoes dramatic changes in protein localization and morphology. The polarity protein, Scribble, is believed to be recruited to the synapse after TCR signaling and, through its potential association with $\beta$ PIX, may recruit Rac1 and Cdc42 to close proximity to GEFs such as Vav. Activated Rac1 and Cdc42 in turn, activate downstream effectors such as WAVE, WASP, and PAK, enabling actin polymerization and thus, changes in morphology. (b) In a migrating T-cell, GTPases regulate actin polymerization to allow for cell moment. At the leading edge of the cell, Cdc42 is activated by the Ras-related protein RAP1a, which in turns activates members of the Par complex. Par3 recruits a RAC GEF, Tiam1, which in turn activates Rac1. Rac1 promotes actin reorganization, thus lamellipodium formation through proteins such as WAVE and Arp2/3. The Par complex also binds and activates the E3 ligase Smurf1. Smurf1 promotes degradation of another GTPase, RhoA, which, in its active form enables actin contractility in cells.

Polarity proteins have been shown to interact extensively with GTPases in T-cells and other cell systems. Following chemokine stimulation, Cdc42 at the leading edge is activated by RAP1A, a Ras-related protein, which activates the Par complex [22]. Tiam1, a Rac GEF, is recruited to the leading edge by Par3 [70, 71] and then activates Rac1, which in turn induces actin nucleation and therefore lamellipodium formation. The Par6-aPKC heterodimer also binds to E3 ligase Smurf1 and activates it. Smurf1 degrades RhoA [72] and therefore reduces actin contractility, resulting in the characteristic dynamic actin polymerization and depolymerization at the leading edge (Figure 1(b)). Scribble and Dlg are found to be asymmetrically distributed in the uropod of migrating T-cells and reduced expression of Scribble and Dlg by shRNA knockdown results in the loss of the uropod and the recruitment of the uropod markers, CD44, and Ezrin. The loss of Scribble also causes abrogation of T-lymphocyte migration [6].

\section{DOCK8: A New Player in T-Cell Polarity}

Apart from the more extensively studied polarity proteins, the protein Dedicator of Cytokinesis 8 (DOCK8), was recently identified as a potential regulator of polarity in immune cells. DOCK8 is a Rho-Rac guanine exchange factor [73] and was first discovered in a screen for binding partners of the Rho GTPase, Cdc42 using a yeast two-hybrid system [74]. The DOCK8 protein has extensive homology to the Ced-5/DOCK180/Myoblast city (CDM) family of proteins [75]. The members of this family of proteins share two conserved domains, DOCK homology regions (DHR) 1 and 2. The important GEF activity is situated in the DHR-2 domain. There are eleven members in the DOCK family [74] but only DOCK180 and DOCK2 have been extensively studied. DOCK2 is required for CD28-mediated Rac activation [76], translocation of the TCR after antigen presentation [77] and lymphocyte migration [78]. DOCK2 


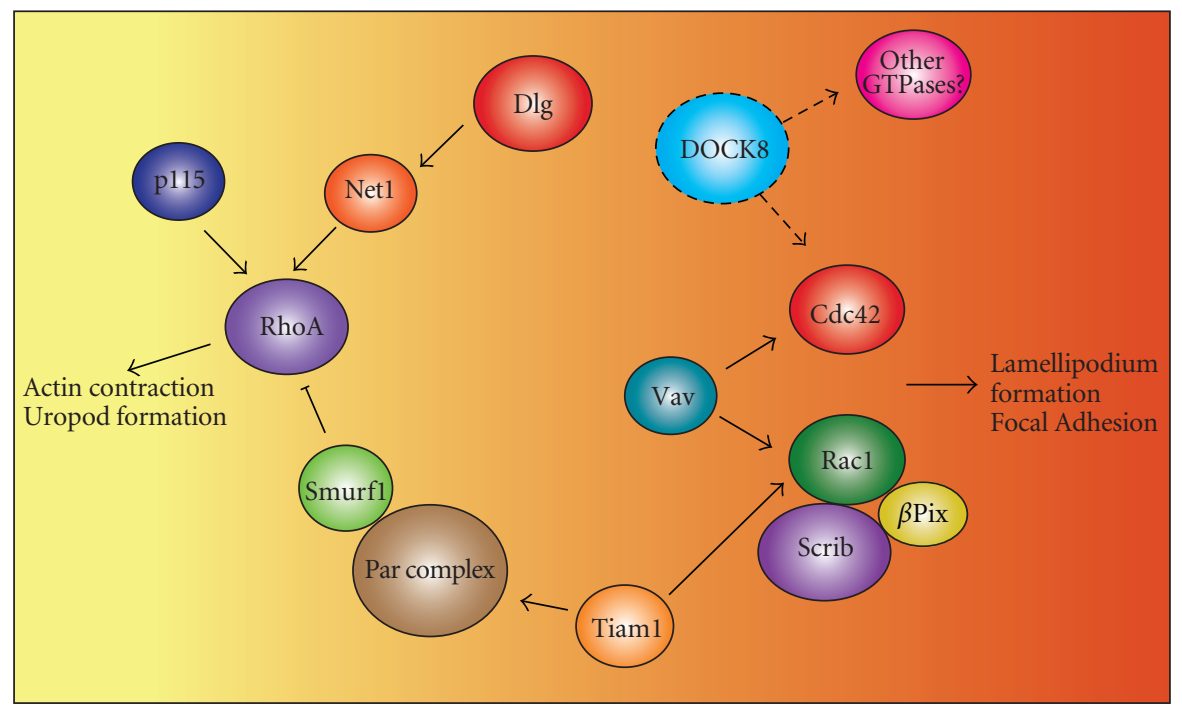

FIGURE 2: GEFs and polarity proteins are important GTPase regulators. GTPases function as switches in cells, controlling a large variety of pathways. They are tightly regulated by Guanine exchange factors (GEFS), GTPase activating proteins (GAPS) and polarity proteins. The recently discovered that GEF, DOCK8, may also be part of this large network. Evidence has shown that it interacts with Cdc42, an important GTPase in the regulation of cell morphology and motility. DOCK8 may also be a regulator of other GTPases that control different cellular functions important for T-cell function.

and DOCK180 are involved in cytoskeletal remodeling [78, 79] and in regulating the activation of Rac [77, 80]. Apart from binding to Cdc42 with high affinity in the yeast twohybrid screen, Ruusala also demonstrated that DOCK8 is localized in the lamellipodia in porcine aortic endothelial cells [74] where extensive actin cytoskeleton remodeling occurs. Therefore, one can speculate that, similar to the other members in the family, DOCK8 is involved in some aspects of actin cytoskeleton regulation. This is reinforced by the fact that DOCK8 serves as a GEF for Cdc42, which is a regulator of cell morphology, migration, and proliferation.

Interestingly, loss-of-function mutations in DOCK8 were recently identified in patients with severe combined immunodeficiency, characterized by repeated bacterial and viral infections [81, 82]. Analysis of patient lymphocytes revealed lower numbers of $\mathrm{CD}^{+}$and $\mathrm{CD} 8^{+} \mathrm{T}$-cells, impairment of T-cell proliferation upon stimulation by anti-CD3 and antiCD28 antibodies, and a moderate decrease in interferon$\gamma($ IFN- $\gamma)$ and tumour necrosis factor- $\alpha($ TNF- $\alpha)[81,82]$. However, the $\mathrm{CD}^{+}{ }^{+} \mathrm{T}$-cells had normal levels of cytotoxic activity as well as extravasation ability [82]. These studies demonstrated, for the first time, that DOCK8 is involved in the regulation of immune cells. DOCK8 has also been speculated to have tumour-suppressor functions, as a number of patients in the study had human papillomavirus infections and cutaneous T-cell lymphoma-leukemia [82].

Using a DOCK8 mutant mouse model, Primurus (pri/ pri), where a point mutation changes a serine to a proline residue in the DHR-2 domain of the DOCK8 protein, Randall et al., [73] have characterized the role of DOCK8 in immune cell function. The pri/pri mutation is thought to break the contact between the DHR-2 domain with Cdc42, and therefore interfere with normal GTP exchange function. Analysis of the pri/pri mice revealed that there are defects in marginal zone B lymphocyte formation as well as in Bcell persistence in the germinal centers. The mutant B-cells are also unable to undergo affinity maturation, resulting in poor longevity in memory-mediated humoral response. The mutation also disrupts the accumulation of ICAM-1 to the pSMAC of the IS [73]. DOCK2 deficient mice also have impaired B-cell migration to lymph nodes but this phenotype is not observed in the pri/pri mice despite the high degree in homology between amino acid sequence between DOCK2 and DOCK8 [74]. This data suggests that DOCK8 may have a specialized role in immune cells.

The severe cutaneous viral infections typical of patients with DOCK8-deficiency in particular, suggest a role for DOCK8 in $\mathrm{CD}^{+}{ }^{+} \mathrm{T}$-cell function. In two separate studies using the pri/pri mouse model, mutation of DOCK8 significantly decreased the number of peripheral naïve $\mathrm{CD} 8^{+} \mathrm{T}$ cells $[83,84]$. Although phenotypically normal, the CD8 ${ }^{+}$ T-cells show delayed proliferation in response to dendritic cells presenting antigen in vitro [83]. Despite this phenotype, DOCK8 deficient mice mount a relatively normal primary immune response to viral infection in vivo, but show significantly impaired persistence and survival of memory $\mathrm{CD}^{+}$T-cells $[83,84]$. Interestingly, this defect correlated with abnormal polarization of LFA- 1 and actin to the immunological synapse formed between naïve $\mathrm{CD}^{+} \mathrm{T}$ cells and antigen-presenting dendritic cells [73] suggesting a polarity defect that results in suboptimal synapse formation. These data, and others $[85,86]$, suggest that the quality of the IS and the downstream signals generated are critical for the development and persistence of memory T-cells. 


\section{Conclusions}

It is now apparent that, similar to polarity of cells of solid tissues [16], polarity of immune cells may be controlled by a dynamic and two-way interaction between polarity proteins and Rho GTPases. The molecular links between the two groups of proteins seem to be predominantly built upon physical interactions between regulators of the Rho GTPases such as the GEFS, and different components of the polarity complexes (Figure 2). As we identify the specific role of each GEF in morphological changes of immune cells, we will begin to elucidate how the polarity proteins influence the localization of each GEF, but at this stage there are many gaps in our knowledge. For instance, new findings regarding DOCK8 clearly demonstrate important roles for this protein in immune cell polarization, but the molecular basis for its polarity is not yet known. In contrast, Tiam1 and $\beta$ PIX have clear roles in T-cell polarity (particularly related to the immunological synapse) and are regulated by known interactions with members of the polarity network. Understanding how each of these players interact to dictate T-cell polarity will be the next big challenge.

\section{References}

[1] M. F. Krummel and I. Macara, "Maintenance and modulation of T cell polarity," Nature Immunology, vol. 7, no. 11, pp. 11431149, 2006.

[2] E. Assemat, E. Bazellières, E. Pallesi-Pocachard, A. Le Bivic, and D. Massey-Harroche, "Polarity complex proteins," Biochimica et Biophysica Acta, vol. 1778, no. 3, pp. 614-630, 2008.

[3] M. C. Montoya, D. Sancho, M. Vicente-Manzanares, and F. Sánchez-Madrid, "Cell adhesion and polarity during immune interactions," Immunological Reviews, vol. 186, pp. 68-82, 2002.

[4] J. Chang, M. Ciocca, I. Kinjyo et al., "Asymmetric proteasome segregation as a mechanism for unequal partitioning of the transcription factor T-bet during T lymphocyte division," Immunity, vol. 34, no. 4, pp. 492-504, 2011.

[5] J. T. Chang, V. R. Palanivel, I. Kinjyo et al., "Asymmetric $\mathrm{T}$ lymphocyte division in the initiation of adaptive immune responses," Science, vol. 315, no. 5819, pp. 1687-1691, 2007.

[6] M. J. Ludford-Menting, J. Oliaro, F. Sacirbegovic et al., "A network of PDZ-containing proteins regulates $\mathrm{T}$ cell polarity and morphology during migration and immunological synapse formation," Immunity, vol. 22, no. 6, pp. 737-748, 2005.

[7] J. L. Round, T. Tomassian, M. Zhang, V. Patel, S. P. Schoenberger, and M. C. Miceli, "Dlgh1 coordinates actin polymerization, synaptic $\mathrm{T}$ cell receptor and lipid raft aggregation, and effector function in T cells," The Journal of Experimental Medicine, vol. 201, no. 3, pp. 419-430, 2005.

[8] J. Oliaro, V. Van Ham, F. Sacirbegovic et al., "Asymmetric cell division of $\mathrm{T}$ cells upon antigen presentation uses multiple conserved mechanisms," The Journal of Immunology, vol. 185, no. 1, pp. 367-375, 2010.

[9] S. Etienne-Manneville, J. B. Manneville, S. Nicholls, M. A. Ferenczi, and A. Hall, "Cdc42 and Par6-PKC $\eta$ regulate the spatially localized association of Dlg1 and APC to control cell polarization," The Journal of Cell Biology, vol. 170, no. 6, pp. 895-901, 2005.
[10] A. B. Jaffe and A. Hall, "Rho GTPases: biochemistry and biology," Annual Review of Cell and Developmental Biology, vol. 21, pp. 247-269, 2005.

[11] M. Nethe and P. L. Hordijk, "The role of ubiquitylation and degradation in RhoGTPase signalling," Journal of Cell Science, vol. 123, no. 23, pp. 4011-4018, 2010.

[12] R. Begum, E. K. M. S. Nur, and M. A. Zaman, "The role of Rho GTPases in the regulation of the rearrangement of actin cytoskeleton and cell movement," Experimental and Molecular Medicine, vol. 36, no. 4, pp. 358-366, 2004.

[13] J. C. Chang, H. H. Chang, C. T. Lin, and S. J. Lo, "The integrin $\alpha 6 \beta 1$ modulation of PI3K and Cdc42 activities induces dynamic filopodium formation in human platelets," Journal of Biomedical Science, vol. 12, no. 6, pp. 881-898, 2005.

[14] C. Vidal, B. Geny, J. Melle, M. Jandrot-Perrus, and M. Fontenay-Roupie, "Cdc42/Rac1-dependent activation of the p21-activated kinase (PAK) regulates human platelet lamellipodia spreading: implication of the cortical-actin binding protein cortactin," Blood, vol. 100, no. 13, pp. 4462-4469, 2002.

[15] M. Parsons, J. Monypenny, S. M. Ameer-Beg et al., "Spatially distinct binding of Cdc42 to PAK1 and N-WASP in breast carcinoma cells," Molecular and Cellular Biology, vol. 25, no. 5, pp. 1680-1695, 2005.

[16] P. O. Humbert, L. E. Dow, and S. M. Russell, "The Scribble and Par complexes in polarity and migration: friends or foes?" Trends in Cell Biology, vol. 16, no. 12, pp. 622-630, 2006.

[17] D. Bilder, M. Schober, and N. Perrimon, "Integrated activity of PDZ protein complexes regulates epithelial polarity," Nature Cell Biology, vol. 5, no. 1, pp. 53-58, 2003.

[18] A. Wodarz and W. B. Huttner, "Asymmetric cell division during neurogenesis in Drosophila and vertebrates," Mechanisms of Development, vol. 120, no. 11, pp. 1297-1309, 2003.

[19] K. J. Kemphues, J. R. Priess, D. G. Morton, and N. Cheng, "Identification of genes required for cytoplasmic localization in early C. elegans embryos," Cell, vol. 52, no. 3, pp. 311-320, 1988.

[20] M. H. Roh, S. Fan, C. J. Liu, and B. Margolis, "The Crumbs3Pals1 complex participates in the establishment of polarity in mammalian epithelial cells," Journal of Cell Science, vol. 116, no. 14, pp. 2895-2906, 2003.

[21] N. B. Adey, L. Huang, P. A. Ormonde et al., "Threonine phosphorylation of the MMAC1/PTEN PDZ binding domain both inhibits and stimulates PDZ binding," Cancer Research, vol. 60, no. 1, pp. 35-37, 2000.

[22] S. Iden and J. G. Collard, "Crosstalk between small GTPases and polarity proteins in cell polarization," Nature Reviews Molecular Cell Biology, vol. 9, no. 11, pp. 846-859, 2008.

[23] P. A. Henkart, "Mechanism of lymphocyte-mediated cytotoxicity.," Annual Review of Immunology, vol. 3, pp. 31-58, 1985.

[24] A. Grakoui, S. K. Bromley, C. Sumen et al., "The immunological synapse: a molecular machine controlling T cell activation," Science, vol. 285, no. 5425, pp. 221-227, 1999.

[25] M. L. Dustin, S. K. Bromley, Z. Kan, D. A. Peterson, and E. R. Unanue, "Antigen receptor engagement delivers a stop signal to migrating T lymphocytes," Proceedings of the National Academy of Sciences of the United States of America, vol. 94, no. 8, pp. 3909-3913, 1997.

[26] A. G. Baxter and P. D. Hodgkin, "Activation rules: the two-signal theories of immune activation," Nature Reviews Immunology, vol. 2, no. 6, pp. 439-446, 2002.

[27] N. D. L. Savage, S. L. Kimzey, S. K. Bromley, K. G. Johnson, M. L. Dustin, and J. M. Green, "Polar redistribution of the 
sialoglycoprotein CD43: implications for T cell function," The Journal of Immunology, vol. 168, no. 8, pp. 3740-3746, 2002.

[28] C. R. F. Monks, B. A. Freiberg, H. Kupfer, N. Sciaky, and A. Kupfer, "Three-dimensional segregation of supramolecular activation clusters in T cells," Nature, vol. 395, no. 6697, pp. 82-86, 1998.

[29] P. A. van der Merwe, "Formation and function of the immunological synapse," Current Opinion in Immunology, vol. 14, no. 3, pp. 293-298, 2002.

[30] K. Burridge and L. Connell, "A new protein of adhesion plaques and ruffling membranes," The Journal of Cell Biology, vol. 97, no. 2, pp. 359-367, 1983.

[31] P. E. Moingeon, J. L. Lucich, C. C. Stebbins et al., "Complementary roles for CD2 and LFA-1 adhesion pathways during T cell activation," European Journal of Immunology, vol. 21, no. 3, pp. 605-610, 1991.

[32] S. Čemerski and A. Shaw, "Immune synapses in T-cell activation," Current Opinion in Immunology, vol. 18, no. 3, pp. 298-304, 2006.

[33] K. D. Mossman, G. Campi, J. T. Groves, and M. L. Dustin, "Altered TCR signaling from geometrically repatterned immunological synapses," Science, vol. 310, no. 5751, pp. 1191-1193, 2005.

[34] K. H. Lee, A. R. Dinner, C. Tu et al., "The immunological synapse balances T cell receptor signaling and degradation," Science, vol. 302, no. 5648, pp. 1218-1222, 2003.

[35] M. L. Dustin and D. Depoil, "New insights into the T cell synapse from single molecule techniques," Nature Reviews Immunology, vol. 11, no. 10, pp. 672-684, 2011.

[36] M. L. Dustin, A. K. Chakraborty, and A. S. Shaw, "Understanding the structure and function of the immunological synapse," Cold Spring Harbor Perspectives in Biology, vol. 2, no. 10, Article ID a002311, 2010.

[37] T. A. Springer, "Traffic signals for lymphocyte recirculation and leukocyte emigration: the multistep paradigm," Cell, vol. 76, no. 2, pp. 301-314, 1994.

[38] S. Ghosh, A. A. Chackerian, C. M. Parker, C. M. Ballantyne, and S. M. Behar, "The LFA-1 adhesion molecule is required for protective immunity during pulmonary Mycobacterium tuberculosis infection," The Journal of Immunology, vol. 176, no. 8, pp. 4914-4922, 2006.

[39] T. A. Springer, "Adhesion receptors of the immune system," Nature, vol. 346, no. 6283, pp. 425-434, 1990.

[40] T. N. Sims and M. L. Dustin, "The immunological synapse: integrins take the stage," Immunological Reviews, vol. 186, pp. 100-117, 2002.

[41] B. A. Lollo, K. W. H. Chan, E. M. Hanson, V. T. Moy, and A. A. Brian, "Direct evidence for two affinity states for lymphocyte function-associated antigen 1 on activated T cells," The Journal of Biological Chemistry, vol. 268, no. 29, pp. 21693-21700, 1993.

[42] D. Li, J. J. Molldrem, and Q. Ma, "LFA-1 regulates CD8 ${ }^{+}$T cell activation via $\mathrm{T}$ cell receptor-mediated and LFA-1-mediated Erk1/2 signal pathways," J Biol Chem, vol. 284, pp. 2100121010, 2009.

[43] J. Jacobelli, S. A. Chmura, D. B. Buxton, M. M. Davis, and M. F. Krummel, "A single class II myosin modulates T cell motility and stopping, but not synapse formation," Nature Immunology, vol. 5, no. 5, pp. 531-538, 2004.

[44] N. G. Dulyaninova, V. N. Malashkevich, S. C. Almo, and A. R. Bresnick, "Regulation of myosin-IIA assembly and Mts1 binding by heavy chain phosphorylation," Biochemistry, vol. 44, no. 18 , pp. 6867-6876, 2005.
[45] S. Russell and J. Oliaro, "Compartmentalization in T-cell signalling: membrane microdomains and polarity orchestrate signalling and morphology," Immunology \& Cell Biology, vol. 84, no. 1, pp. 107-113, 2006.

[46] R. Xavier, S. Rabizadeh, K. Ishiguro et al., "Disc large (Dlg1) complexes in lymphocyte activation," The Journal of Cell Biology, vol. 166, no. 2, pp. 173-178, 2004.

[47] A. Ivetic and A. J. Ridley, "Ezrin/radixin/moesin proteins and Rho GTPase signalling in leucocytes," Immunology, vol. 112, no. 2, pp. 165-176, 2004.

[48] A. V. Miletic, D. B. Graham, K. Sakata-Sogawa et al., "Vav links the $\mathrm{T}$ cell antigen receptor to the actin cytoskeleton and $\mathrm{T}$ cell activation independently of intrinsic guanine nucleotide exchange activity," PLoS One, vol. 4, no. 8, Article ID e6599, 2009.

[49] K. D. Fischer, K. Tedford, and J. M. Penninger, "Vav links antigen-receptor signaling to the actin cytoskeleton," Seminars in Immunology, vol. 10, no. 4, pp. 317-327, 1998.

[50] S. Audebert, C. Navarro, C. Nourry et al., "Mammalian scribble forms a tight complex with the $\beta$ PIX exchange factor," Current Biology, vol. 14, no. 11, pp. 987-995, 2004.

[51] R. Ahmed, M. J. Bevan, S. L. Reiner, and D. T. Fearon, "The precursors of memory: models and controversies," Nature Reviews Immunology, vol. 9, no. 9, pp. 662-668, 2009.

[52] S. Feau and S. P. Schoenberger, "Immunology: ex uno plura," Science, vol. 323, no. 5913, pp. 466-467, 2009.

[53] S. C. Jameson and D. Masopust, "Diversity in T cell memory: an embarrassment of riches," Immunity, vol. 31, no. 6, pp. 859-871, 2009.

[54] S. M. Kaech and E. J. Wherry, "Heterogeneity and cell-fate decisions in effector and memory $\mathrm{CD}^{+} \mathrm{T}$ cell differentiation during viral infection," Immunity, vol. 27, pp. 393-405, 2007.

[55] O. Leavy, "Lymphocyte activation: unequal inheritance initiates T-cell diversity," Nature Reviews Immunology, vol. 7, no. 4, p. 247, 2007.

[56] D. R. Littman and H. Singh, "Asymmetry and immune memory," Science, vol. 315, no. 5819, pp. 1673-1674, 2007.

[57] D. M. Catron, L. K. Rusch, J. Hataye, A. A. Itano, and M. K. Jenkins, "CD4 ${ }^{+} \mathrm{T}$ cells that enter the draining lymph nodes after antigen injection participate in the primary response and become central-memory cells," The Journal of Experimental Medicine, vol. 203, no. 4, pp. 1045-1054, 2006.

[58] S. L. Reiner, F. Sallusto, and A. Lanzavecchia, "Division of Labor with a workforce of one: challenges in specifying effector and memory T cell fate," Science, vol. 317, no. 5838, pp. 622-625, 2007.

[59] S. M. Russell, "Determination of T-cell fate by dendritic cells: a new role for asymmetric cell division?" Immunology \& Cell Biology, vol. 86, no. 5, pp. 423-427, 2008.

[60] J. Betschinger and J. A. Knoblich, "Dare to be different: asymmetric cell division in Drosophila, C. elegans and vertebrates," Current Biology, vol. 14, no. 16, pp. R674-R685, 2004.

[61] E. C. Lai and V. Orgogozo, "A hidden program in Drosophila peripheral neurogenesis revealed: fundamental principles underlying sensory organ diversity," Developmental Biology, vol. 269, no. 1, pp. 1-17, 2004.

[62] M. Vicente-Manzanares, J. R. Cabrero, M. Rey et al., "A role for the Rho-p160 Rho coiled-coil kinase axis in the chemokine stromal cell-derived factor- $1 \alpha$-induced lymphocyte actomyosin and microtubular organization and chemotaxis," The Journal of Immunology, vol. 168, no. 1, pp. 400-410, 2002. 
[63] M. Nieto, J. M. R. Frade, D. Sancho, M. Mellado, C. MartinezA, and F. Sánchez-Madrid, "Polarization of chemokine receptors to the leading edge during lymphocyte chemotaxis," The Journal of Experimental Medicine, vol. 186, no. 1, pp. 153-158, 1997.

[64] J. M. Serrador, J. L. Alonso-Lebrero, M. A. del Pozo et al., "Moesin interacts with the cytoplasmic region of intercellular adhesion molecule- 3 and is redistributed to the uropod of T lymphocytes during cell polarization," The Journal of Cell Biology, vol. 138, no. 6, pp. 1409-1423, 1997.

[65] J. V. Small, T. Stradal, E. Vignal, and K. Rottner, "The lamellipodium: where motility begins," Trends in Cell Biology, vol. 12, no. 3, pp. 112-120, 2002.

[66] S. Etienne-Manneville and A. Hall, "Rho GTPases in cell biology," Nature, vol. 420, no. 6916, pp. 629-635, 2002.

[67] M. A. del Pozo, M. Vicente-Manzanares, R. Tejedor, J. M. Serrador, and F. Sánchez-Madrid, "Rho GTPases control migration and polarization of adhesion molecules and cytosketetal ERM components in T lymphocytes," European Journal of Immunology, vol. 29, no. 11, pp. 3609-3620, 1999.

[68] W. E. Allen, G. E. Jones, J. W. Pollard, and A. J. Ridley, "Rho, Rac and Cdc42 regulate actin organization and cell adhesion in macrophages," Journal of Cell Science, vol. 110, supplement 6, pp. 707-720, 1997.

[69] J. H. Lee, T. Katakai, T. Hara, H. Gonda, M. Sugai, and A. Shimizu, "Roles of p-ERM and Rho-ROCK signalling in lymphocyte polarity and uropod formation," The Journal of Cell Biology, vol. 167, no. 2, pp. 327-337, 2004.

[70] X. Chen and I. G. Macara, "Par-3 controls tight junction assembly through the Rac exchange factor Tiam1," Nature Cell Biology, vol. 7, no. 3, pp. 262-269, 2005.

[71] H. Zhang and I. G. Macara, "The polarity protein PAR-3 and TIAM1 cooperate in dendritic spine morphogenesis," Nature Cell Biology, vol. 8, no. 3, pp. 227-237, 2006.

[72] H. R. Wang, Y. Zhang, B. Ozdamar et al., "Regulation of cell polarity and protrusion formation by targeting RhoA for degradation," Science, vol. 302, no. 5651, pp. 1775-1779, 2003.

[73] K. L. Randall, T. Lambe, A. Johnson et al., "Dock8 mutations cripple B cell immunological synapses, germinal centers and long-lived antibody production," Nature Immunology, vol. 10, no. 12, pp. 1283-1291, 2009.

[74] A. Ruusala and P. Aspenström, "Isolation and characterisation of DOCK8, a member of the DOCK180-related regulators of cell morphology," FEBS Letters, vol. 572, no. 1-3, pp. 159-166, 2004.

[75] K. Reif and J. G. Cyster, "The CDM protein DOCK2 in lymphocyte migration," Trends in Cell Biology, vol. 12, no. 8, pp. 368-373, 2002.

[76] H. Jiang, F. Pan, L. M. Erickson et al., "Deletion of DOCK2, a regulator of the actin cytoskeleton in lymphocytes, suppresses cardiac allograft rejection," The Journal of Experimental Medicine, vol. 202, no. 8, pp. 1121-1130, 2005.

[77] T. Sanui, A. Inayoshi, M. Noda et al., "DOCK2 regulates Rac activation and cytoskeletal reorganization through interaction with ELMO1," Blood, vol. 102, no. 8, pp. 2948-2950, 2003.

[78] Y. Fukui, O. Hashimoto, T. Sanui et al., "Haematopoietic cell-specific CDM family protein DOCK2 is essential for lymphocyte migration," Nature, vol. 412, no. 6849, pp. 826$831,2001$.

[79] H. Hasegawa, E. Kiyokawa, S. Tanaka et al., "DOCK180, a major CRK-binding protein, alters cell morphology upon translocation to the cell membrane," Molecular and Cellular Biology, vol. 16, no. 4, pp. 1770-1776, 1996.
[80] E. Kiyokawa, Y. Hashimoto, S. Kobayashi, H. Sugimura, T. Kurata, and M. Matsuda, "Activation of Rac1 by a Crk SH3binding protein, DOCK180," Genes and Development, vol. 12, no. 21, pp. 3331-3336, 1998.

[81] K. R. Engelhardt, S. McGhee, S. Winkler et al., "Large deletions and point mutations involving the dedicator of cytokinesis 8 (DOCK8) in the autosomal-recessive form of hyper-IgE syndrome," The Journal of Allergy and Clinical Immunology, vol. 124, no. 6, Article ID e1284, pp. 1289-1302, 2009.

[82] Q. Zhang, J. C. Davis, I. T. Lamborn et al., "Combined immunodeficiency associated with DOCK8 mutations," The New England Journal of Medicine, vol. 361, no. 21, pp. 20462055, 2009.

[83] K. L. Randall, S. S. Chan, C. S. Ma et al., "DOCK8 deficiency impairs CD8 $\mathrm{T}$ cell survival and function in humans and mice," The Journal of Experimental Medicine, vol. 208, no. 11, pp. 2305-2320, 2011.

[84] T. Lambe, G. Crawford, A. L. Johnson et al., "DOCK8 is essential for T-cell survival and the maintenance of $\mathrm{CD}^{+} \mathrm{T}$ cell memory," European Journal of Immunology, vol. 41, no. 12, pp. 3423-3435, 2011.

[85] A. Scholer, S. Hugues, A. Boissonnas, L. Fetler, and S. Amigorena, "Intercellular adhesion molecule-1-dependent stable interactions between $\mathrm{T}$ cells and dendritic cells determine CD8 ${ }^{+}$T cell memory," Immunity, vol. 28, no. 2, pp. 258270, 2008.

[86] E. Teixeiro, M. A. Daniels, S. E. Hamilton et al., "Different T cell receptor signals determine $\mathrm{CD}^{+}$memory versus effector development," Science, vol. 323, no. 5913, pp. 502-505, 2009. 


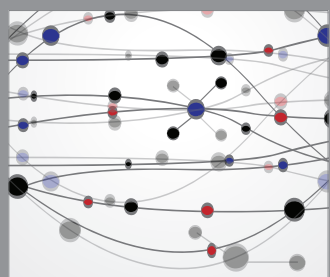

The Scientific World Journal
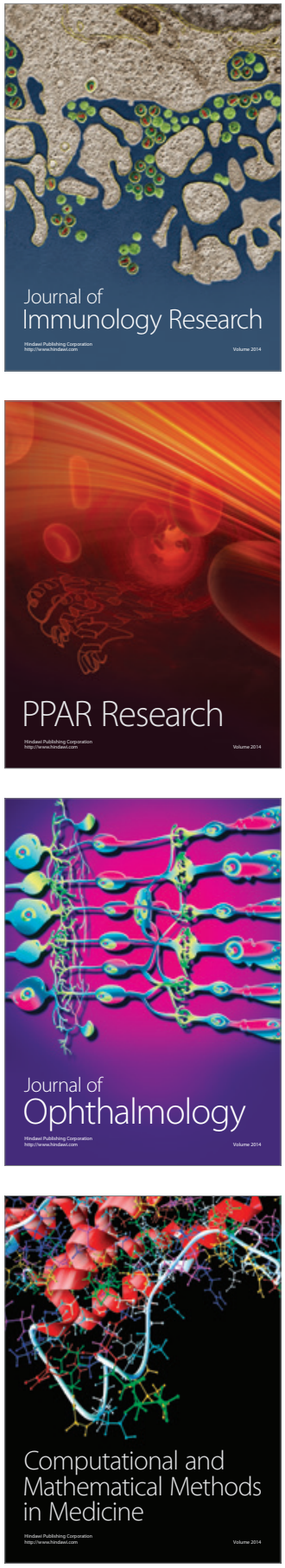

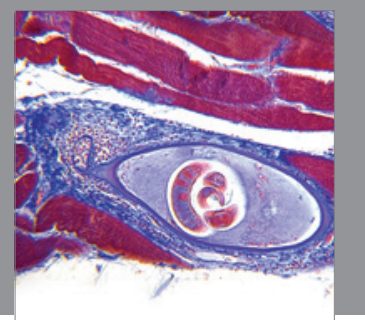

Gastroenterology

Research and Practice
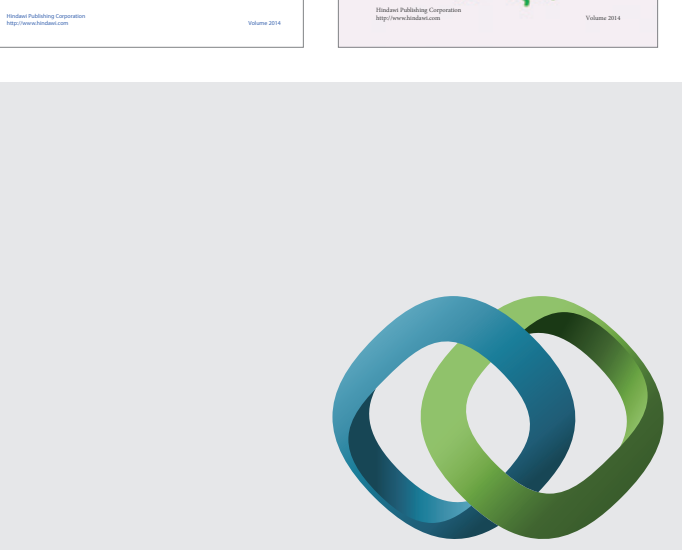

\section{Hindawi}

Submit your manuscripts at

http://www.hindawi.com
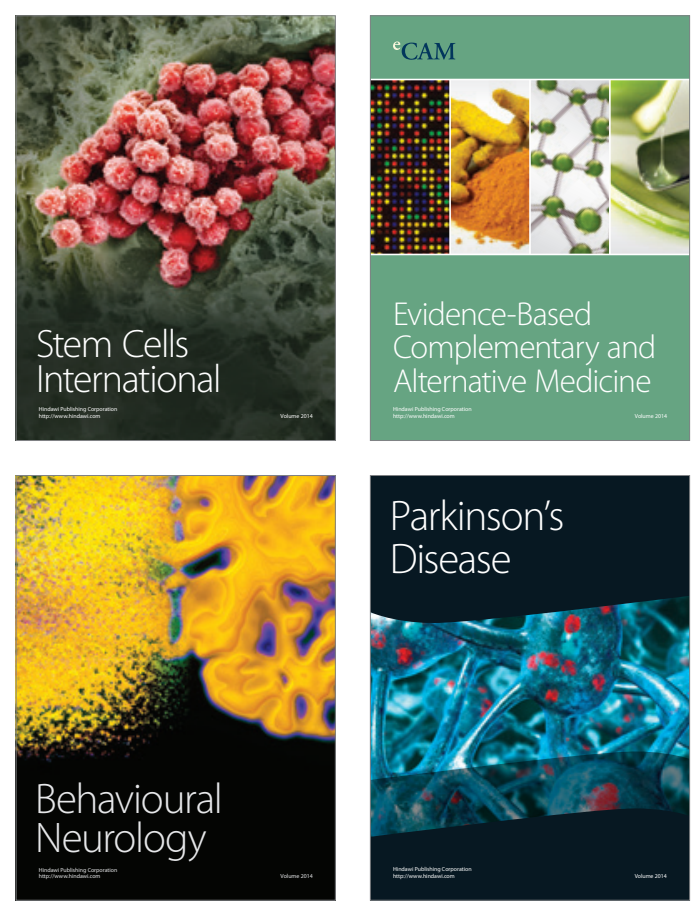

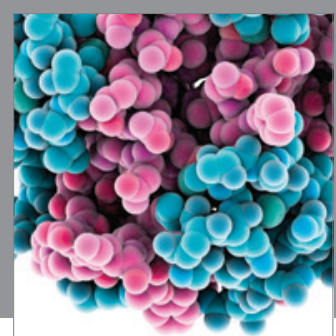

Journal of
Diabetes Research

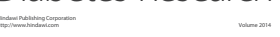

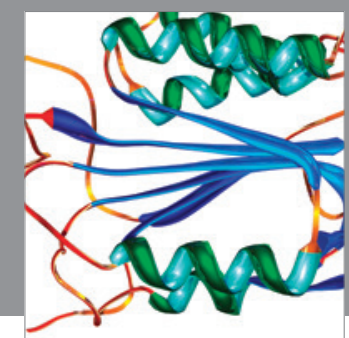

Disease Markers
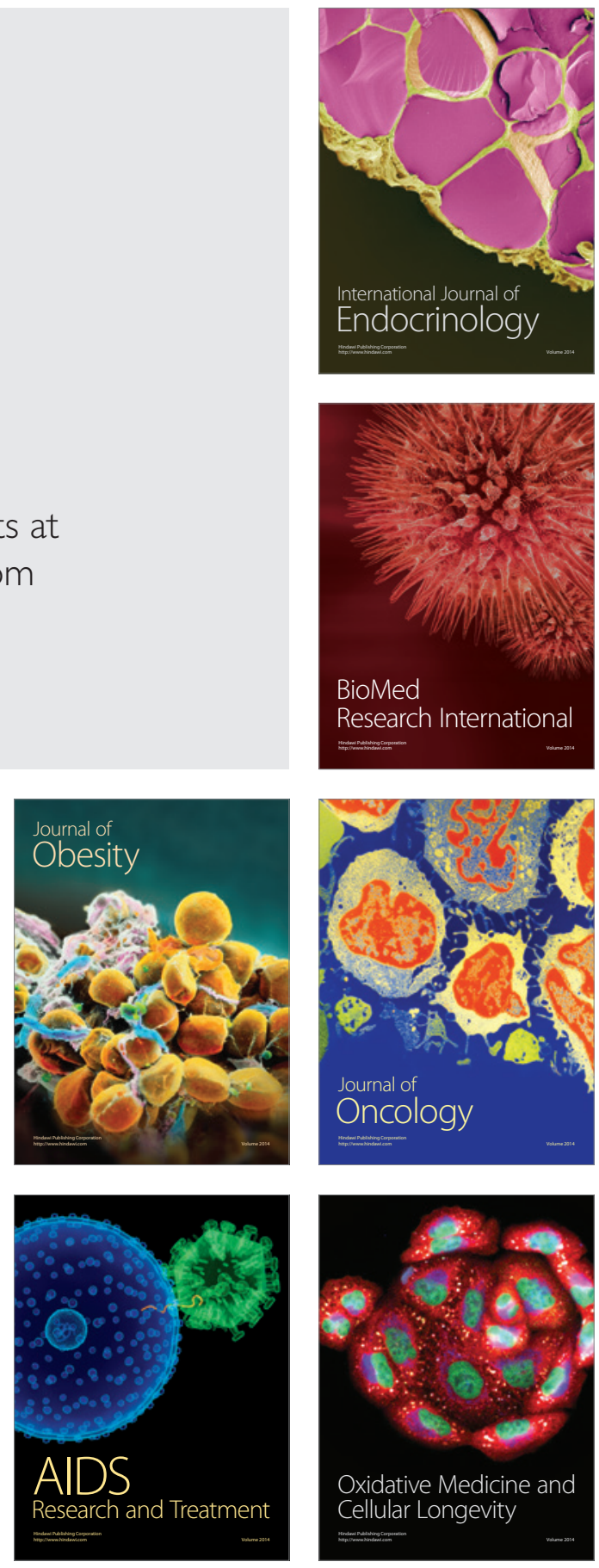